\title{
POLÍTICAS PÚBLICAS EN LAS CIENCIAS BIOLÓGICAS Y LA BIOTECNOLOGÍA.
}

\author{
PONENCIA DEL ECUADOR \\ RED LATINOAMERICANA DE CIENCIAS BIOLÓGICAS (RELAB)
}

\section{Dr. Claudio Cañizares}

Servicio de Hematología y Banco de Sangre.

Hospital Andrade Marín IESS, Quito

\author{
I Plan Nacional \\ de Ciencia y Tecnología
}

Las políticas de las ciencias y la tecnología han sido estructuradas por la Secretaría Nacional de Ciencias y Tecnología en el I Plan Nacional de Investigación Científica y Tecnológica que se puso en marcha en 1996 y se encuentra en vigencia.

En dicho plan se establece que el órgano rector es la Secretaría Nacional de Ciencia y Tecnología (SENACYT), dependiente de la Vicepresidencia de la República y el operativo es la Fundación Nacional de Ciencia y Tecnología (FUNDACYT) como organismo autónomo ejecutivo regido por una Junta Directiva presidida por el Secretario Nacional de Ciencia y Tecnología y con vocales representantes de las Universi- dades, Comunidad Científica y la Empresa Privada.

La finalidad es promover y conseguir un avance cualitativo $\mathrm{y}$ rápido en la incorporación nacional de la ciencia y la tecnología a la dinámica de la sociedad ecuatoriana.

El objetivo general es fortalecer y consolidar el Sistema Nacional de C\&T, robusteciendo la oferta, estimulando la demanda de C\&T y promoviendo la articulación del Sistema. Para ello se plantean los siguientes objetivos a corto y mediano plazo:

1. Formación de nuevos investigadores de alto nivel

2. Crear las condiciones que favorezcan y estimulen la Investigación en las unidades e instituciones ejecutoras de la I $8 \mathrm{DD}$. 
3. Establecimiento de estándares de evaluación y acreditación de investigadores e instituciones.

4. Asignación de recursos económicos para proyectos de $I \& D$, con procedimientos de evaluación y seguimiento

5. Dotación de infraestructura física mínima, dando preferencia a las - unidades de I\&D de excelencia.

6. Promoción y apoyo al establecimiento de nuevas instituciones que vayan integrando el sistema, calificándolas de acuerdo a la oferta y demanda.

7. Conformación de redes de oferta y demanda y su conexión con redes mundiales.

8. Fortalecimiento de los servicios de información y documentación.

9. Promoción del uso, demanda y generación de tecnología e innovación en el sector productivo.

10. Definición de políticas estatales consistentes con la C\&T.

11. Revisión y actualización de la legislación y práctica de la propiedad intelectual.

12. Promoción de la transformación y mejoramiento de la educación superior, a través de recursos para proyectos de excelencia.

13. Dotación de recursos para el funcionamiento y progreso del Sistema con una programación plurianual y evaluaciones periódicas.
Estos objetivos conseguirán el fortalecimiento por etapas consiguiendo las finalidades propuestas de:

1. Priorización de la C\&T como política de Estado.

2. Fortalecimiento estructural y de la actividad de sistema nacional de C\&T

3. Fortalecimiento de la oferta de C\&T.

4. Promoción de la demanda de C\&T.

5. Articulación del Sistema de C\&T.

Una vez hecho el diagnóstico del sistema de C\&T se establecieron las áreas prioritarias de acción, que son las siguientes para la primera etapa todavía en vigencia y que fueron tomadas en cuenta para la aplicación del Proyecto BID/FUNDACYT, que ha sido ejecutado.

a) Biomedicina;

b) Alimentos;

c) Recursos naturales y medio ambiente;

d) Materias primas y minerales;

e) Ingeniería y procesos industriales.

Además se establecen una serie adicional de áreas y temáticas, algunas que representan una precisión o acotación de las cinco grandes áreas citadas, que son las siguientes:

a) Ciencias naturales básicas: química, física, biología y matemáticas; 
b) Biotecnología vegetal de especies cultivadas o silvestres;

c) Biodiversidad y ecosistemas naturales;

d) Sistemas ecológicos y toxicología ambiental;

e) Clima, prevención de catástrofes naturales y recursos hídricos;

f) Salud y nutrición;

g) Vivienda a bajo costo;

h) Selección, adaptación y generación de tecnologías para la enseñanza;

i) Tecnologías productivas horizontales: automatización, disminución de impactos ambientales, disminución de insumos (materias primas, agua, energía);

j) Ingeniería química, con especial referencia a la petroquímica $y$ farmacología;

k) Tecnologías apropiadas para sectores productivos medianos y pequeños;

1) Turismo sostenible;

m) Ciencias sociales: economía, sociología, antropología.

Hay que indicar que este plan de desarrollo en ciencia y tecnología financiado con un préstamo BID de 30 millones de dólares y con una contrapartida nacional de 10 millones ha terminado y actualmente se está trabajando en un nuevo préstamos que permita la continuación de las acciones una vez que han sido evaluadas y reajustadas.
Existe un análisis diagnóstico en cuanto a las áreas. Indicaremos lo relacionado a aquellas del sector de ciencias biológicas y biotecnología que se señalan en el plan nacional.

\section{Biología:}

Dado el poco desarrollo de la investigación biológica básica en el Ecuador, se puede afirmar que la proporción/participación en el PIB, ocupación e incidencia en otros sectores es limitada $y$ no cuantificable.

Sin embargo, la ventaja comparativa nacional de mayor importancia radica en la alta biodiversidad del país que ofrece ventajas comparativas con la investigación en otras partes del mundo. Además el avance en la investigación científica de esta área brindará capacidad de solucionar problemas de índole socio-económico relacionado a la aplicación de tecnologías científicas.

El estudio de los aspectos de la biodiversidad del país brindará ventajas competitivas a nivel nacional. Se logrará publicaciones internacionales que introduzcan patentes, disminuyendo la dependencia técnico-científica extranjera y permitiendo la competitividad científica a nivel internacional.

\section{Los problemas son:}

- Limitado apoyo económico.

- Falta de reconocimiento de la investigación a nivel de la sociedad.

- Ausencia de programas de doctorado e investigación. 
- Carencia de grupos de trabajo estables.

- No hay evaluación objetiva y real de las ciencias biológicas básicas.

- Limitado impacto de las publicaciones. Muy pocas constan en los índices de publicaciones internacionales. A nivel local se publican en revistas o publicaciones esporádicas institucionales.

- Se desconoce de patentes registradas en esta área.

\section{Necesidades:}

- Capacitación y perfeccionamiento del personal.

- Apoyo económico para la infraestructura física y de equipamiento $y$ para la accesibilidad a fuentes bibliográficas.

- Reconocimiento por parte del Estado y la sociedad de la importancia de esta área como fuente para el desarrollo.

- Incrementar la población científica activa en ciencias biológicas básicas.

- Generar conocimientos en la materia como fundamento necesario $e$ ineludible para la investigación aplicada en biomedicina, farmacología, biotecnología.

- Fortalecer las líneas de investigación en esta área.

\section{Ciencias Agrícolas}

Producción Animal

Se orienta casi en su totalidad al mercado interno de carnes y cueros. El sector agropecuario representa un 13.16 del PIB.

Se mantiene la ventaja comparativa de la producción animal nacional, en tanto y en cuanto los productos importados (carne fresca refrigerada, lácteos, huevos...) no tienen mayor demanda en el consumo interno.

El Instituto Nacional de Investigaciones Agropecuarias ha obtenido éxitos en la transferencia de tecnología, así como capacitación de personal. En el caso de las universidades hay esfuerzos investigativos, pero las tesis de grado no son conocidas fuera de las mismas.

\section{Problemas:}

- La sanidad animal tiene bajos niveles de servicios veterinarios. Frecuentes epidemias, ausencia de producción local de fármacos veterinarios.

- Predominio de la ganadería extensiva (pastos) sin que se añadan concentrados proteínicos.

- Restricciones en la reproducción y mejoramiento genético.

\section{Necesidades:}

- Fortalecer la capacidad de C\&T nacional relacionada con la producción animal y mejorar la calidad, productividad y eficiencia del sector agropecuario proveedor de los insumos alimenticios. Consolidando las líneas de investigación en temas específicos que ofrezcan respuestas a la problemática nacional. Estimular 
los recursos humanos especialmente en campos como la ingeniería genética, la biotecnología.

- Utilización de un conjunto de instrumentos y maquinarias que tecnifiquen el conjunto de procesos del sector, sistemas que cualifiquen el desenvolvimiento de sus actividades, dotación de recursos financieros.

\section{Forestal}

Representa uno de los recursos renovables de importancia para el país por su aspecto económico y por la preservación ambiental. Las áreas forestales se localizan especialmente en la región oriental y noroccidental, donde se explotan recursos para la industria maderera, papelera, farmacéutica, etc. Su aporte al PIB es importante del 3.66\%. Sin embargo hay una alta importación de pulpa y papel. Hay un alto empleo de mano de obra rural.

Está incrementándose la exportación de flores.

\section{Problemas}

- Ausencia de políticas de conservación, manejo y explotación de los recursos forestales.

- Deforestación por ampliación del área agrícola y la tala.

- Deterioro de la calidad de aguas que sirve para el uso humano y la supervivencia de especies acuáticas.

- Falta de estadísticas confiables.

- Baja rentabilidad y largos ciclos de producción de las plantaciones.

\section{Necesidades:}

- Estructurar un programa de investigación de largo alcance para optimizar los recursos humanos, infraestructura y financiamiento que ofrezcan soluciones a los problemas del sector.

- Propender a la conservación y aprovechamiento racional de los recursos forestales y naturales para reducir la deforestación controlándolas y con programas de reforestación.

\section{Recursos pesqueros}

Es importante. Hay que diferenciar entre la pesquería y la acuicultura. La pesquería representa el $6 \%$ de la exportaciones la acuicultura es más importante por su crecimiento en las exportaciones de camarón.

\section{Problemas:}

- En la pesquería hay insuficiente información respecto a la captura de especies.

- Inadecuadas políticas sectoriales y inadecuada capacidad institucional que generan conflictos entre los pescadores, turismo, ecología.

- Sobreexplotación de ciertas especies, lo cual pone en peligro las especies y disminución de las capturas.

- Restricciones de otros países para la importación. 


\section{Necesidades:}

- Adecuación y ampliación de los sistemas sobre el potencial pesquero y las capturas.

- Mejoramiento de la pesca artesanal.

- Diversificación de las especies para el consumo del mercado.

- Investigación biológica sobre crecimiento, reproducción, hábitat, alimentación de las especies de acuerdo a las prioridades establecidas por el Instituyo Nacional de Pesca.

\section{Acuicultura}

Es un sector relativamente nuevo. En el Ecuador ha crecido importantemente y se ha situado en el segundo producto de exportación del país, sin embargo el problema de las enfermedades del camarón ha afectado seriamente a este sector en los últimos años.

\section{Problemas:}

- Falta de crédito.

- Disminución progresiva del precio por aumento en la oferta por otros países.

- Exagerado incremento en los precios de los insumos.

- Enfermedades de los camarones.

- Impacto sobre las áreas de los manglares.

\section{Necesidades:}

- Falta de suministros de larvas, semillas, alevines.
- Enfermedades (bacterias, protozoarios, virus).

- Variaciones climáticas drásticas (El Nińo y Anti-Niño).

- Falta de recursos económicos para realizar investigaciones sostenidas.

- Enforzamiento de políticas para controlar la tala de manglares.

\section{Alimentos}

Este sector se orienta al mercado interno, en su mayor parte por consumo directo provenientes de las áreas agrícola, pecuaria y acuícola. Aportan con un $21.8 \%$ del PIB.

De exportación hay congelados, secos y elaborados. Los productos más importantes para la exportación son de la Costa el banano, cacao, café. En los últimos años se han incorporado espárragos y frutas de la Sierra.

\section{Problemas:}

- Desarrollo incipiente de la industria alimenticia.

- Dependencia de tecnología extranjera.

- Baja integración productiva entre el sector industrial y agrícola.

- Falta de control y esfuerzos para la mayor obtención de productos adecuados.

- Escasa experiencia técnica y limitado financiamiento.

- Falta de gestión de calidad total y bajo nivel tecnológico. 


\section{Necesidades:}

- Impulsar la biotecnología en este sector daría variedad y mejor calidad en los productos que asegure las necesidades alimenticias de la población, dado que hay graves problemas de desnutrición.

- Se estima que los años 2000 la biotecnología tendrá el mayor impacto en el sector agrícola.

- Estímulo en las políticas nacionales para impulsar cultivos de productos agrícolas que han decaído, y que están siendo importados cada vez más, como trigo, cebada, etc, y de productos nativos con valor medicinal y alimenticio.

- Desarrollar la rama alimentos procesados, apoyando a los pequeños $y$ medianos productores que requieren de conocimiento y servicios de C\&T para elevar niveles de producción y competitividad.

\section{Biodiversidad}

Por la ubicación y características geográficas del país se sitúa entre los más ricos en cuanto a biodiversidad de flora, fauna y microorganismos.

\section{Problemas:}

- Débiles derechos de propiedad, interferencia gubernamental con las fuerzas del mercado.

- Escasa conciencia sobre la importancia y valor de la naturaleza.

- Mantenimiento de estructuras económicas y sociales que atentan contra el espacio ecológico y productividad biológica.

- Inadecuados instrumentos jurídicos para la conservación de la biodiversidad.

\section{Necesidades:}

- Las ciencias biológicas básicas tienen un peso específico en el desarrollo de esta área, sin embargo dado el incipiente papel de C\&T en el país su participación es muy limitada.

- La biotecnología y la manipulación de los recursos genéticos tienen una gran importancia por tanto deben ser estimulados a través de centros de formación e investigación en estas áreas.

- Se requiere contribuir a la conservación y desarrollo sostenible de los recursos biológicos en sus diferentes manifestaciones.

\section{PROGRAMA DE DESARROLLO CIENTIÍFICO Y TECNOLÓGICO PROYECTO BID/FUNDACYT 874/OC-EC}

El plan nacional de C\&T ha sido ejecutado entre 1966 y 2001, fundamentalmente a través del Préstamo BID 874/OC-EC. Para los efectos de la ejecución del Programa se han definido las siguientes áreas prioritarias: 
Biomedicina

Alimentos

Materias Primas y Minerales

Recursos Naturales y Medio

Ambiente

Ingeniería y Procesos Industriales

1.- Proyectos de investigación: 36 aprobados. Duración hasta 48 meses.

Biomedicina

Alimentos y Agricultura

8

Recursos Naturales

9

Meteorología y Sísmica 2

Medio Ambiente

2

Materias Primas y

Minerales

Ingeniería y Procesos

Industriales
Materias Primas y

Minerales

Ingeniería y Procesos

Doctorados en

el Exterior:

Biomedicina

Alimentos

Medio Ambiente

Materias Primas

Ingeniería y Procesos

\section{Información}

Red Ecuatoriana de Información Científica y Tecnológica

Instituciones

Participantes

5. Innovación y

6

Modernización de

Empresas

FONDOS NACIONALES

Biología

Polímeros

Recursos Hidricos

Ingeniería Estructural

y Sísmica

\section{3.- Formación}

Maestrías en el Exterior: 61

Biomedicina

Alimentos

Medio Ambiente

\section{1.- Promoción de C\&T}

Centros En estudio

Interactivos

de C\&T

Ferias

En ejecución.

Juveniles

de C\&T

Mejoramiento "Compulab" 


\author{
de la Enseñanza En ejecución \\ en C\&T \\ Encuentros de En ejecución \\ C\&T \\ Empresas- Universidad
}

Formación de En ejecución

Redes de

Investigadores

\section{2.- Eventos}

Nacionales:

Ciencias de la Salud

Ciencia y Tecnología

de Alimentos

Recursos Naturales

y Medio Ambiente

Materias Primas y Minerales

Ingeniería y Procesos Industriales

Ciencias Básicas:

Física, Matemáticas,

Química, Biología y Geología

Ciencias de la Computación

Prevención de Desastres

Técnicas de Construcción

Ciencias Sociales

\section{Exterior}

Programa Iberoamericano de Ciencia y Tecnología (CYTED).

Red Latinoamericana de Ciencias Biológicas (RELAB).

Apoyo para asistencia a eventos.

\section{3.- Publicaciones}

Libros, Revistas, Centro de Información.

Problemas encontrados en el I Plan de C\&T.

1.- Reformulación de proyectos múltiples veces.

2.- Baja calidad científica del país, lo cual se reflejaba sobre todo en la formulación de proyectos.

3.- Divorcio entre los centros de I\&D y la realidad nacional.

4.- Sector privado sin esfuerzo apropiado para la innovación tecnológica.

5.- Financiamiento limitado para los estudios en el exterior que no permiten que los que hayan terminado la maestría sigan con el doctorado. No crédito para las familias de los becarios.

6.- Áreas prioritarias sin postulantes.

\section{PROGRAMA DE CIENCIA Y TECNOLOGÍA}

\section{PROGRAMA DE \\ INNOVACIÓN PARA EL DESARROLLO (PID) En trámite}

Objetivos para el desarrollo de la ciencia, la tecnología e innovación (CTI).

Consolidar el sistema nacional de innovación.

Contribuir a la solución de algunos problemas sociales más significativos $y$ 
mejorar la calidad de vida de la población a través de la investigación científica.

Mejorar la competitividad internacional de los principales sectores productivos a través de la incorporación del conocimiento y la tecnología.

Incrementar la base de recursos humanos de alta calificación necesarios para el desarrollo C\&T.

Asegurar la continuidad de los desarrollos científicos y el uso eficiente de la infraestructura de investigación y tecnologías de información del país.

\section{Conceptualización:}

El PID estará organizado básicamente en programas nacionales temáticos de CTI.

Las áreas no están aún determinadas, ni en número ni en tema, pero quedarán establecidas por sectores involucrados en el transcurso de los próximos meses, a través de talleres que se organizan para el efecto.

A estos programas se agregará un Programa de Promociones del Conocimiento C\&T que involucrará a los actores que no se encuentran incluidos en ninguna de las otras áreas seleccionadas.

Las modalidades de financiamiento (componentes) propuestas para atender programas nacionales temáticos dentro del PID, serán:

Investigación científica, que incluye financiamiento para:

- Proyectos de investigación.

- Proyectos de interés nacional.
Innovación tecnológica, que incluye financiamiento para:

- Proyectos de innovación competitiva.

- Proyectos de innovación precompetitiva.

Fortalecimiento y crecimiento de capacidades, que incluye financiamiento para:

- Becas institucionales

- Cursos y pasantías

- Redes, seminarios y talleres

- Postgrados nacionales

- Fortalecimiento y articulación del sistema de innovación para desarrollar estudios relevantes, sistemas de información, divulgación, difusión $\mathrm{y}$ fortalecimiento institucional.

El PID prevé un financiamiento de US\$ 25 millones que provendrá de un nuevo crédito del BID.

El dimensionamiento inicial propuesto de los componentes del PID es:

- $35 \%$ para investigación científica

- $35 \%$ para innovación tecnológica

- $18 \%$ para fortalecimiento y creación de capacidades

- $7 \%$ para fortalecimiento y articulación del sistema de innovación

- $5 \%$ para gastos administrativos

Cada Programa contará con un Comité de Programa integrado por empresarios, científicos y representantes del gobierno para definir los lineamien- 
tos estratégicos en los cuales se insertarán las actividades de apoyo a la ciencia, tecnología e innovación definidos en el PID. Buscará disminuir las diferencias entre el sector privado y científico en materia de desarrollo C\&T.

En el PID, a diferencia del I Plan de $C \& T$, se optará por la modalidad de cofinanciamiento no reembolsable de los proyectos de innovación del sector empresarial y se diseñarán esquemas de tramitación que permitan dar respuesta ágil y efectiva a los empresarios.

Este modelo permitirá tener una visión integrada y articulada de todas las oportunidades y acciones en un determinado campo, facilitando las colaboraciones entre los actores.

\section{FONDOS NACIONALES}

Se seguirán aplicando en programas de:

\section{Promoción}

Eventos

Publicaciones

Capacitación

En el momento actual el nuevo préstamo del BID no se ha hecho efectivo, de manera que la situación programática de FUNDACYT no ha variado y continua funcionando con los fondos propios.

\section{FUENTES}

POLIÍTICAS DE LAS CIENCIAS $Y$ LA TECNOLOGIA. I Plan Nacional de Investigación Científica y Tecnológica. Ecuador 1966. SENACYT FUNDACYT.

EXCERTA 1997. FUNDACYT. Ecuador.

II PROGRAMA NACIONAL DE CIENCIA Y TECNOLOGÍA. 2000 SENACYT FUNDACYT

PROGRAMA DE INNOVACIÓN PARA EL DESARROLLO-PID2002. SENACYT FUNDACYT 